\title{
Experimental evidence of chiral symmetry breaking in crystallization from primary nucleation
}

\author{
Cristobal Viedma* \\ Departamento de Cristalografia y Mineralogía, Facultad de Geología, Universidad Complutense, 28040 Madrid, Spain
}

\begin{abstract}
Chiral symmetry breaking in stirred crystallization of sodium chlorate $\left(\mathrm{NaClO}_{3}\right)$ is believed to occur via nucleation of a single chiral "mother crystal" that produces a new generation of crystals which inherit its chirality. We here report results of stirred crystallizations resulting in massive "instantaneous" primary nucleation and total symmetry breaking. The results of these experiments suggest that the current explanation for chiral symmetry breaking via nucleation of a single "mother crystal" is not adequate and that the mechanism to explain the chiral symmetry breaking in crystallization remains as an open question.
\end{abstract}

Keywords: A1. Chirality; A1. Secondary nucleation; A2. Symmetry breaking

\section{Introduction}

Chiral symmetry breaking occurs when a physical or chemical process that does not have preference for the production of one or other enantiomer spontaneously generates a large excess of one of the two enantiomers. Spontaneous chiral symmetry breaking is of particular interest to physics, chemistry and biology. The fact that biologically relevant molecules such as amino acids and nucleic acids exist only as one of the two enantiomers is a fascinating example. What mechanisms are responsible for spontaneous chiral generation from a totally symmetry state? An

*Tel.: + 34-913944868; fax: + 34913944872.

E-mail address: Viedma@geo.ucm.es (C. Viedma). experimental example of such spontaneous chiral symmetry breaking was reported by Kondepudi et al. [1] for the stirred crystallization of $\mathrm{NaClO}_{3}$. The achiral molecules of $\mathrm{NaClO}_{3}$ crystallize in enantiomeric chiral crystals. Though an aqueous solution of this compound is not optically active, the chiral crystals are. The levo 1 and dextro d rotatory crystals can be easily identified with a pair of polarizers because crystal color changes, when the polarized are crossed, on rotating the analyzer clockwise or anticlockwise depending on the handedness of the crystal [1,2]. Symmetry breaking in this case occurs in a simple way: if $\mathrm{NaClO}_{3}$ is crystallized from an unstirred solution, statistically equal numbers of levo and dextro crystals are obtained. If the crystallization is performed while the solution is constantly stirred, more than $99 \%$ 
of the crystals have the same handedness. In any particular run, the dominance of 1 and $d$ crystals is random so each enantiomer can dominate with equal probability in different solutions. Kondepudi [1] suggested that the most important factor in this chiral symmetry breaking is secondary nucleation. In a primary nucleation crystals are generated from the solution itself. On the other hand, secondary nucleation is the process by which a "mother crystal" generates secondary crystals at a fast rate if the solution is stirred; in the case of $\mathrm{NaClO}_{3}$, a first 1 or d "mother crystal" generates new crystals with similar chirality in a few minutes. The sequence of events is as follows [3,4]: a single crystal randomly nucleates at some time by primary nucleation and begins to grow. When the crystal reaches a critical size it begins to produce secondary nuclei, which have the same chirality as their "mother". These are quickly dispersed throughout the system. The rapid production of secondary nucleation can be due to hydrodynamic shear or due to collision with the stirrer. This causes the solution to drop in concentration to a value at which the rate of production of new nuclei of opposite handedness from primary nucleation is virtually zero. The result of this crystallization process is the generation of crystals with the same handedness (chiral symmetry breaking). As noted by McBride and Carter [5], it is remarkable that only one initial crystal dominates the entire system, not simply in principle or through an occasional chance, but virtually always and in every stirred crystallization. To check the experimental validity of this mechanism we have conducted a systematic study of the behavior of primary nucleation in $\mathrm{NaClO}_{3}$ solutions. Our objective was to determine if primary nucleation, without a previous "mother crystal", is capable or not of producing chiral symmetry breaking in a stirred solution.

\section{Materials and methods}

We prepared a set of 10 solutions of $\mathrm{NaClO}_{3}$ saturated at $80^{\circ} \mathrm{C}\left(10 \mathrm{~g}\right.$ of $\mathrm{NaClO}_{3}$ per $6.8 \mathrm{~g} \mathrm{H}_{2} \mathrm{O}$ every one). The solutions were hermetically sealed and stirred for $2 \mathrm{~h}$ at $90^{\circ} \mathrm{C}$ to ensure complete desolution of the solute and then, without opening, cooled to room temperature $\left(20^{\circ} \mathrm{C}\right)$. Despite the very high supersaturation level, the solutions remained metastable without crystallizing. Then, every solution vessel was put on an electromagnetic stirrer, and we begin to stir suddenly and very rapidly (approximately1000 rpm). Almost instantaneously the solution became cloudy (white milky aspect) with the formation of micro-crystals. The crystals thus obtained were allowed to grow for 2 days.

\section{Results and discussion}

The rate of nucleation was estimated to be in the range of $700,000900,000$ crystals in every particular solution. Crystals, with a size of about $20 \mu \mathrm{m}$, were sub-idiomorphic and develop a cubic habit with a $\left\{\begin{array}{lll}0 & 0 & 0\end{array}\right.$ form. The chirality of random samples in every glass vessel was identified using a polarizing microscope. In samples of the same solution $100 \%$ of crystals were of the same handedness.

The primary nucleation rate, from a supersaturated solution, is given by the following [6]:

$\boldsymbol{J}=\Gamma \exp \left(\frac{-\delta \sigma^{3} \Omega^{2}}{k^{3} T^{3}\left[\ln \left(C / C_{\mathrm{s}}\right)^{2}\right]}\right)$,

where $\delta$ is a shape factor, $\sigma$ is the nucleus-solution interfacial tension and $\Omega$ is the volume of one growth unit in the nucleus. The pre-exponential factor $\Gamma$ is related to the rate at which the nucleus can grow to a supercritical size. $T$ is the temperature and $k$ is Boltzmann's constant. $S$ is the supersaturation ratio $C / C_{\mathrm{s}}$, where $C_{\mathrm{s}}$ is the equilibrium concentration of the solution in equilibrium with the crystalline phase and $C$ is the actual concentration of the solution.

From this expression, and others similarly used to express the nucleation-supersaturation relation, it is clear that the nucleation rate is a direct function of supersaturation ratio $C / C_{\mathrm{s}}$ which is the most important feature in symmetry breaking processes [7]. The equation also indicates the 
catastrophic nature of the primary nucleation process: there is a critical supersaturation below which the nucleation rate is very low and above which it becomes extremely high [8]. In a typical symmetry breaking experiment $[1,9]$, the initial concentration of a stirred solution is below the equilibrium concentration. In order to crystallize the solution is necessary to cause its supersaturation by either evaporation or cooling. In either case the process is performed very slowly. This means that as the solution evaporates or cools down, the concentration increases until it slowly reaches the supersaturation region to nucleate. Under these conditions, and from expression (1), we can expect an initial low nucleation rate at the low supersaturation level. In fact this occurs and the rate of nucleation obtained by video observations in one of this usual symmetry breaking experiments was found to be in the range of 12 nuclei per minute for an entire solution of $100 \mathrm{ml}$ [7]. In the same paper the authors calculated a nucleation rate of $0.2 \mathrm{nuclei} / \mathrm{min}$ when $\left(C / C_{\mathrm{s}}\right)=1.001$ and 2 nuclei/ min when $\left(C / C_{\mathrm{s}}\right)=1.04$. These low values of nucleation rate support the explanation of a unique first "mother crystal" that generates secondary crystals with the same chirality in a few minutes. However, in our case we start the stirring experiments from a very high supersaturation level $\left(C / C_{\mathrm{s}}>1.5\right.$ and $60^{\circ} \mathrm{C}$ of supercooling). Therefore, from a theoretical point of view (expression (1)), we should be dealing with a process of catastrophic primary nucleation, with a very high nucleation rate. This, in fact, is what we observe: hundreds of thousands of crystals are generated almost instantaneously in a catastrophic event. In this primary nucleation experiment, we find also a total symmetry breaking phenomenon: $100 \%$ of crystals have the same chirality in every solution. Therefore, at least in this case, the idea of a single first "mother crystal" inducing secondary chiral nucleation, is not appropriate to account for symmetry breaking process. Moreover, Qian and Botsaris [9] have shown that, in an artificially seeded solution of $\mathrm{NaClO}_{3}$, as the supersaturation of solution increases, the probability that secondary nuclei generated have the same chirality as the mother seed decreases. They found experimentally that in the range of $36^{\circ} \mathrm{C}$ of supercooling, nearly $100 \%$ of the secondary crystals generated in this stirred seeded solution had the same chirality than the initial seed; however, if the supercooling exceeded $6^{\circ} \mathrm{C}$, the fraction of secondary crystals with the same handedness of the seed was much smaller and it varied randomly. They concluded that this occurs because, in their experimental conditions, at $6^{\circ} \mathrm{C}$ of supercooling the solution is at or very close to the critical supercooling temperature for spontaneous primary nucleation and that only at moderate supercooling, avoiding primary nucleation, the effect of the seeding can be real in the symmetry breaking process. This necessity of a low supersaturation level for symmetry breaking in the hypothesis of a first "mother crystal", is admitted by Kondepudi et al. [4] when explaining the experimental results of Martin et al. [10]. These results indicate that a very rapid crystallization leading to formation of large number of crystal nuclei, should result in an equal number of 1 and d crystals (no symmetry breaking). In our experiments however the supercooling considerably exceeds the limit of $6^{\circ} \mathrm{C}\left(60^{\circ} \mathrm{C}\right)$. The crystallization process is very rapid (almost instantaneous), and the symmetry breaking is $100 \%$. This clearly indicates that we are not dealing with secondary nucleation phenomena from a previously generated chiral crystal but a real primary nucleation involving symmetry breaking that cannot be explained in terms of secondary nucleation. Our results do not necessarily contradict the results of Kondepudi and others however if we change the concept of first "mother crystal" for a concept of first "mother nucleation". This is because after a primary chiral nucleation occurs, to survive as unique handedness, processes of chirally autocatalytic secondary nucleation must be present in order to avoid a new primary nucleation with different chirality. A stirred solution of $\mathrm{NaClO}_{3}$ is an evolutionary system, in which the first chiral nucleation takes the control and amplifies, by secondary nucleation, the initial symmetry breaking, which in turn becomes total. But the real chiral symmetry breaking occurs in the first steps of the primary nucleation, whereas secondary nucleation only amplifies the symmetry breaking. 


\section{Conclusions}

The results of our experiments suggest that the current explanation for chiral symmetry breaking via nucleation of a single "mother crystal" may explain some cases but not all, and as such, the mechanism to explain the chiral symmetry breaking in crystallization remains as an open question.

\section{References}

[1] D. Køndepudi, R. Kaufman, N. Singh, Science 250 (1990) 975.

[2] B. Martin, A. Tharrington, X.-L. Wu, Phys. Rev. Lett. 77 (1996) 2826.

[3] D. Køndepudi, K.L. Bulløck, J.A. Digits, P.D. Yarbør๑ugh, J. Am. Chem. Søc. 117 (1995) 401.

[4] T. Buhse, D. Durand, D. Køndepudi, J. Laudadie, S. Spilker, Phys. Rev. Lett. 84 (2000) 4405.

[5] J.M. McBride, R.L. Carter, Agew. Chem. 30 (1991) 293.

[6] A.E. Nielsen, Kinetics $\bullet$ Precipitatiøn, Per

[7] D. Køndepudi, K.L. Bulløck, J.A. Digits, J.K. Hall, J.M. Miller, J. Am. Chem. S•c. 115 (1993) 10211

[8] R.-Y. Qian, D. Bøtsaris, Chem. En. Sci. 52 (1997) 3429.

[9] R.-Y. Qian, D. Botsaris, Chem. Eng. Sci. 53 (1998) 1745.

[10] B. Martin, A. Tharringten, X.-L. Wu, Phys. Rev. Lett. 77 (1996) 2826 . 